\title{
Massive stars in colliding wind systems: the GLAST perspective
}

\author{
Anita Reimer \& Olaf Reimer
}

Stanford University, HEPL \& KIPAC, Stanford, CA 94305, USA

\begin{abstract}
Colliding winds of massive stars in binary systems are considered as candidate sites of high-energy non-thermal photon emission. They are already among the suggested counterparts for a few individual unidentified EGRET sources, but may constitute a detectable source population for the GLAST observatory.

The present work investigates such population study of massive colliding wind systems at high-energy gamma-rays. Based on the recent detailed model (Reimer et al. 2006) for non-thermal photon production in prime candidate systems, we unveil the expected characteristics of this source class in the observables accessible at LAT energies. Combining the broadband emission model with the presently cataloged distribution of such systems and their individual parameters allows us to conclude on the expected maximum number of LAT-detections among massive stars in colliding wind binary systems.
\end{abstract}

Keywords: gamma-ray - Mass loss and stellar winds - Star counts, distribution, and statistics - Main-sequence: early-type stars - Binary and multiple stars - gamma-ray sources

PACS: 95.85.Pw; 97.10.Me; 97.10.Yp; 97.20.Ec; 97.80.-d; 98.70.Rz

\section{INTRODUCTION}

Massive stars are hot, high-mass and luminous $\left(\sim 10^{5 \ldots 6} L_{\odot}\right)$ stellar systems that possess supersonic winds $\left(V_{\mathrm{W}, \infty} \sim\right.$ several $1000 \mathrm{~km} / \mathrm{s})$ and accompagnied with a large mass loss rate $\left(\dot{M} \sim 10^{-6 \ldots-4} M_{\odot} / \mathrm{yr}\right)$. From more than a dozen galactic Wolf-Rayet (WR) binary systems non-thermal radio emission has been observed [e.g., 1], and this is interpreted as synchrotron radiation from a relativistic electron distribution. As a consequence inverse Compton (IC) scattering in the dense photospheric radiation fields of the stars as the presumably dominant leptonic emission process [8, 13] is guaranteed to produce high-energy photons. Observational indications for massive stars systems being high-energy sources comes from population studies of the still unidentified EGRET sources: correlations with various massive star populations have been found [e.g.,23], although their unambiguous identification must await instruments with significantly improved source localization capabilities (e.g. GLAST). The site of particle acceleration may be the instability driven shocks inside the winds [29], shocked wind collision regions of multiple systems [8, 5] or the termination shock [25]. The following work concerns colliding winds (CWs) of WR-binary systems. Its main characteristics [8] has been meanwhile impressively confirmed by radio as well as X-ray observations [see e.g., 7, 15, for the nearby WR 147 system]: The collision of supersonic winds is creating a region of hot shocked gas that is separated by a contact discontinuity, and a forward and reverse shock follows. Its stagnation point is defined by the balance of the wind momenta (assuming homogeneous winds). The observation of synchrotron radiation indicates that the winds are permeated by magnetic fields supposedly originating from the massive star's surface. Estimates for surface magnetic field strengths range from below $B_{s}=100 \mathrm{G}$ (e.g. [12]) up to $\sim 10^{4} \mathrm{G}[9]$. In the following we fix this value to a reasonable $100 \mathrm{G}$, unless stated otherwise, and use the magnetic rotator theory (e.g. [28]) to estimate the field strength at the CWR. Typically $>\mathrm{mG}$ or higher field strengths are expected, assumed to be constant throughout the emission region. Note that because the stellar target photons for IC scattering arrive at the collision region from a preferred direction, the full angular dependence of the cross section has to be taken into account (e.g. [22]). This leads to anisotropy effects like the emitted flux and cutoff energy being dependent on the sight line into the wind [see e.g., 20]. In binary systems the maximum flux level will be reached at phases where the WR- is behind the OB-star along the line-of-sight. Photon absorption due to $\gamma \gamma$ pair production may affect the spectrum at energies above $\sim 70 \mathrm{GeV}$ in $\mathrm{CW}$ systems.

The most up-to-date catalogs of galactic WR- [26, 27] and OB-stars [10] contains more than 220 and 370 sources, respectively, with a detected binary fraction of $\sim 40-50 \%$ [26]. More than $30 \%$ are located in clusters and associations. Its spatial distribution reflects the spiral arm structure of our Galaxy ([26] and references therein). The total number of WR-(systems) in the Galaxy has been estimated to 8000 [17], total OB-star number counts in our Galaxy may reach values as high as 60000 [19]. This is at least an order of magnitude larger than the number of all presum- 
ably galactic, but still unidentified $\gamma$-ray sources detected to date. How many of those massive WR-binary systems will GLAST's LAT be detecting at most? By combining a realistic physical source model with the statistics offered by the star catalogs we aim to answer this question in the following. The result will have implications for the chosen approach to probe the existence of WR-binaries in the the GLAST data. Whereas spatial coincidences are expected given the number of cataloged Wolf-Rayet stars and the anticipated number of $\gamma$-ray source detections in the Galactic Plane above GLAST sensitivity, this study provides a prior for the size of a plausible gamma-ray bright WR-binary population as well as the individuals to be preferably investigated among the population.

\section{SAMPLE SELECTION AND PARAMETERS}

The construction of the sample for our study starts with the galactic WR-catalog [26, 27], selecting all 88 binary systems. For more than a dozen of those sources the nominal shock location falls below the star's photosphere if windmomentum balance determines the shock location, making the CW scheme an unsuitable setting for those. These are excluded in the following. Because of the distance squared dilution factor, close-by sources have a higher probability to be detected. Indeed, our statistical study shows that sources beyond $\sim 3 \mathrm{kpc}$ have a negligible chance to become a LAT source. Hence a distance cutoff in our sample was chosen at $4 \mathrm{kpc}$, which excludes a further 42 systems. Finally, a $\gamma$-ray flux estimate on the basis of model simulations requires at least the knowledge of the shock location, estimated from its period or stellar separation and wind momenta of the participating stars, to provide reasonable estimates for the photon and field density at the shock. More than 10 sources have no period assigned, and hence are excluded from this study. We end up considering 21 galactic WR-binaries for potential LAT detectability.

For estimating the maximum possible flux in LAT's energy range we consider in the following the IC component only as the most dominant $\gamma$-ray production process, and use the Reimer et al. (2006) WR-binary model. The most important system parameters that impact the flux estimate significantly are the photospheric luminosity and temperature of the stars, their masses and momentum loss rates, their stellar separation and system distance. Only the distribution of the two latter ones shows an appreciable spread whereas all others have rather narrow ones, owing to the definition of a massive star. System parameters have been derived from [26, 11, 14, 24]. Because we're seeking the maximum number of likely LAT-sources among the WR-binary population, we allow the theoretically highest possible acceleration rate $\left(\dot{E} \propto E c / R_{L}\left(V_{W} / c\right)^{2}, R_{L}=\right.$ Larmor radius) and evaluate the $\gamma$-ray output at a phase of maximum ICflux. The system eccentricity $e$ is known for only half a dozen sources in the considered sample with an average $e \approx 0.2$ and the highest being $e \approx 0.9$. In the following we assume $e=0$, and evaluate then the resulting $\gamma$-ray flux uncertainty using extreme eccentricities. The same applies to the surface field, fixed at $B_{s}=100 \mathrm{G}$ now. An inclination angle of $90^{\circ}$ is used for unknown system inclinations. Particle number and energy conservation arguments apply for the electron energy injection, as outlined in [20]. An particle injection spectrum $\propto E^{-2}$ is used throughout this work. With these parameters we calculate the maximum flux expected at $>100 \mathrm{MeV}$ for all sample sources using the model of [20].

\section{RESULTS}

We consider a source to be detectable by the LAT if the maximum photon energy reaches at least the LAT energy range, and if the photon flux $>100 \mathrm{MeV}$ is above LAT's sensitivity. For the latter we use an estimated factor 5 higher sensitivity level as published for LAT's high-latitude sensitivity $1,2 \times 10^{-8} \mathrm{~cm}^{-2} \mathrm{~s}^{-1}$, to account the fact that for the massive star systems being located preferentially in the Galactic Plane. Among the considered sample we find 6-7 WRbinaries to be detectable by the LAT in an one-year exposure, of which three are positional coincident with EGRET unidentified sources. All but one (for WR 70 only an upper limit in the radio band exists) turn out to be non-thermal radio emitters, and tend to be preferentially very-long-period binaries (see Fig. 1). The ratio of the wind momenta together with the stars' separation determines the distance of the shock $x_{\mathrm{OB}}$ above the photosphere. The resulting distribution of the the shock-star separation (see Fig. 1) highlights the preference of LAT-detectible sources to possess larger values of $x_{\mathrm{OB}}$. This is plausible in the light of the severe IC-losses causing a low energy cutoff in the electron spectrum if the shock is located too deep in the dense photospheric radiation field, and thus inhibiting GeV photon production in shorter-period binaries. Finally, we find that only close-by systems $(<1 \mathrm{kpc})$ have a high chance to be

1 http://www-glast.slac.stanford.edu/software/IS/glast_lat_performance.htm 

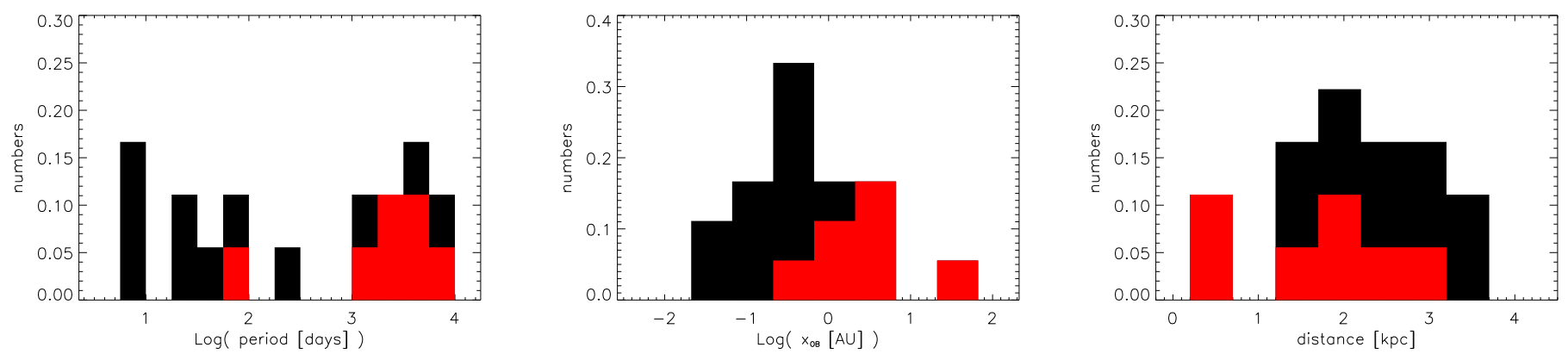

FIGURE 1. Normalized source number distribution of period (left), shock-star distance $x_{\mathrm{OB}}$ (middle) and source distance (right) of LAT-detectable WR-binaries (red/grey) from the source sample in comparison to all sample sources (red/grey and black).

detected by the LAT, whereas WR-binaries located beyond $\sim 1 \mathrm{kpc}$ but up to $\sim 3 \mathrm{kpc}$ have a significantly reduced detection probability (see Fig. 1).

To estimate the robustness of our result in view of unavoidable uncertainties in the parameter values, we vary each parameter (distance $d_{L}$, stellar separation $D_{\mathrm{WR}-\mathrm{OB}}$, surface magnetic field $B_{*}$, mass loss rates $\dot{M}_{\mathrm{WR}}$, wind velocities $V_{\infty, \mathrm{WR}, \mathrm{OB}}$, stellar temperature $T_{\text {eff }}$ and luminosity $\left.L_{\mathrm{bol}}\right)$ and deduce the resulting number of LAT-detectable sources after each change. Specifically we alter period, $d_{L}, B_{*}, \dot{M}_{\mathrm{WR}, \mathrm{OB}}, V_{\infty, \mathrm{WR}, \mathrm{OB}}, T_{\text {eff }}$ and $L_{\mathrm{bol}}$ by a factor $2,2,10,10,2$, 0.2 and 2 respectively. A variation of the system eccentricity to the extreme possible values adds a further factor $\pm_{0.1}^{1.9}$ uncertainty to the stellar separation. As a result we find 6-7 systems in our sample are LAT-detectable with a total uncertainty of $\pm_{5}^{2}$, leading to the conclusion that the expected maximum number of detectable WR-binaries in a one-year GLAST-LAT exposure may be as high as eight, but could be as low as only one source from this population.

Identification procedures may substancially benefit from physically motivated estimates of the expected source density of the respective population, like suggested here for the case of WR-binaries. Further parameter studies will include their spatial distribution, flux variability and orbital periodicity, spectral features, and signatures obtained at other wavelengths (see [21] for a discussion applied to massive star systems).

\section{REFERENCES}

1. Abbott, D.C., Bieging, J.H., Churchwell, E. \& Torres, A.V. 1986, ApJ, 303, 239 (1986)

5. Bykov, A.M.\& Fleishman, G.D., MNRAS, 255, 269 (1992)

7. Dougherty, S.M., ASP Conference Proc. “Interacting winds from massive stars”, 260, 287 (2002)

8. Eichler, D. \& Usov, V., ApJ, 402, 271 (1993)

9. Igance, R., Cassinelli, J.P. \& Bjorkman, J.E., ApJ, 505, 910 (1998)

10. Maíz-Apellániz, J., Walborn, N.R., Galué, H.A., et al., ApJS, 151, 103 (2004)

11. Markova, N., Puls, J., Scuderi, S., et al., A\&A 440, 1133 (2005)

12. Mathys , G. in: "Variable and Non-spherical Stellar Winds in Luminous Hot Stars", eds. Wolf, B. et al., Lecture Notes in Physics, 523, 95 (1999)

13. Mücke, A. \& Pohl, M., in: "Interacting winds from massive stars", ASP conf. series, 260, 355 (2002)

14. Nugis, T. \& Lamers, H.J.G.L.M., $A \& A, \mathbf{3 6 0}, 227$ (2000)

15. Pittard, J.M., Stevens, I.R., Williams, P.M. et al., A\&A, 388, 335 (2002)

17. Prantzos, N. \& Casse, M., ApJ, 307, 324 (1986)

19. Reed, B.C., $A J, \mathbf{1 2 0}, 314$ (2000)

20. Reimer, A., Pohl, M. \& Reimer, O., A\&A, 644, 1118 (2006)

21. Reimer, A., Reimer, O. \& Pohl, M., Astrophysics \& Space Science, in press (2007); astro-ph/0611647

22. Reynolds, A.P., ApJ, 256, 13 \& 38 (1982)

23. Romero, G.E., Benaglia, P., Torres, D.F., $A \& A, \mathbf{3 4 8}, 868$ (1999)

24. Schaerer, D. \& Maeder, A., $A \& A$, bf 263, 129 (1992)

25. Völk, H.J. \& Forman, M., ApJ, 253, 188 (1982)

26. van der Hucht, K.A., New Astronomy Reviews, 45, 135 (2001)

27. van der Hucht, K.A., $A \& A, 458,453$ (2006)

28. Weber, E.J. \& Davis, L.Jr., ApJ, 148, 217 (1967)

29. White, R.L., ApJ, 289, 698 (1985)

30. Williams P.M., van der Hucht, K.A., Pollock, A.M.T., et al., MNRAS, 243, 662 (1990)

31. Williams P.M., Dougherty, S.M., Davis, R.J., et al., MNRAS, 289, 10 (1997) 